\title{
Strategic Bidding Behaviors in Nondecreasing Sponsored Search Auctions
}

\author{
Chen-Kun Tsung, ${ }^{1}$ Hann-Jang Ho, ${ }^{2}$ and Sing-Ling Lee ${ }^{1}$ \\ ${ }^{1}$ Institute of Computer Science and Information Engineering, National Chung Cheng University, No. 168, Section 1, University Road, \\ Min-Hsiung Township, Chia-yi County 62102, Taiwan \\ ${ }^{2}$ Department of Applied Digital Media, WuFeng University, No. 117, Section 2, Chiankuo Roadd, Min-Hsiung Township, \\ Chia-yi County 62153, Taiwan
}

Correspondence should be addressed to Hann-Jang Ho; hannjangho@gmail.com

Received 23 September 2013; Accepted 9 October 2013

Academic Editor: Teen-Hang Meen

Copyright (C) 2013 Chen-Kun Tsung et al. This is an open access article distributed under the Creative Commons Attribution License, which permits unrestricted use, distribution, and reproduction in any medium, provided the original work is properly cited.

\begin{abstract}
To realize the specific results in the sponsored search auctions, most advertisers submit particular bid prices. The bidding behaviors with specific purposes are called as the strategic bidding. However, some strategic bidding behaviors will result in negative effects, such as the elimination of the equilibrium and the payment increase for some advertisers. The bidding behaviors with negative results are termed as the vindictive bidding. We survey four strategic bidding behaviors which include a rational bidding and three vindictive bidding strategies. In this paper, we study the relationship between the effects resulted by the vindictive bidding and the valuations of the vindictive advertisers. In our experiments, the search engine provider (SEP) is benefited by all vindictive bidding behaviors, and the increment of the SEP's revenue is proportional to the degree of the vindictiveness. Bidding vindictively without sacrificing the own utility improves the advertiser's utility with high probability. Moreover, we observe that the SEP's revenue is improved by the following situations. First, the vindictive advertiser with low valuation in the keywords with high market value results in more SEP's revenue than that in the keywords with low market value. The second case is to raise the bidding competition between advertisers.
\end{abstract}

\section{Introduction}

When an Internet user submits a keyword query to the SEP, such as Google or Yahoo, the user will receive a web page including the search results and some advertisements. Combining the search service and the advertising is called the sponsored search auction (SSA) $[1,2]$ or the keyword auction [3-5]. Figure 1 is an example of the SSA service provided by Yahoo. The user queries the keyword Taiwan tour agency and then receives the web page. The center area includes the search results while the area with red box is the advertisements recommended by Yahoo. The advertising area includes some slots, and each slot displays some information of an advertisement including a title, a hyperlink connected to the source web page, and a brief description. Because the recommended advertisements are related to the input keywords, the advertising information is useful for the readers. Therefore, most Internet users accept the advertisements recommended by the SSAs.
Most SSAs consider the pay-per-click and the generalized second price (GSP) as the pricing mechanisms $[6,7]$. The payper-click indicates that each advertiser pays for advertising only when the Internet user clicks the advertisement. The GSP charges each winner the essential price of winning the slot; that is, each payment equals to the next-ranked bid value rather than the claimed bid price. The pricing mechanism improves the advertising effectiveness, and consequently many advertisers prefer to promote their products via the SSAs. Therefore, the SSAs become major incoming of the SEPs $[6,7]$.

Some advertisers attempt to realize the specific outcomes by submitting particular bid values, and that is called as the strategic bidding. For example, to realize the outcomes with maximum utility, $\mathrm{Bu}$ et al. proposed the forwardlooking bidding strategy [4], and Cary et al. introduced the balanced bidding strategy [5]. Both bidding strategies guarantee the equilibrium outcome (The equilibrium is 


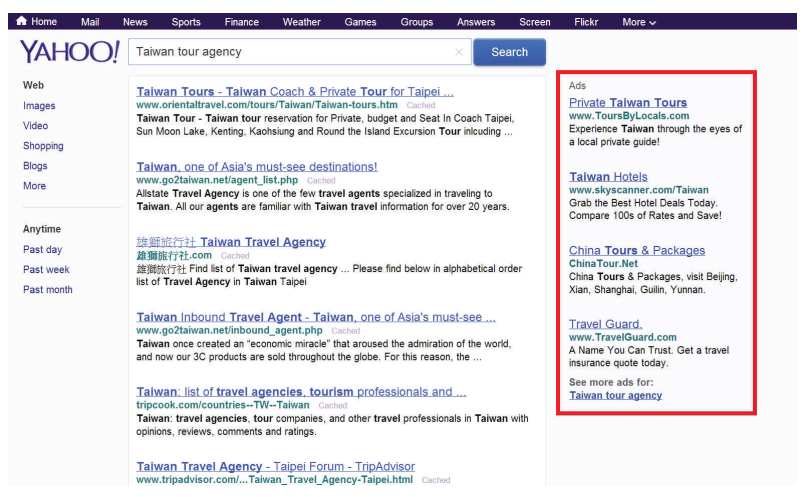

FIgURE 1: An example of the SSA provided by Yahoo! with the keyword phrase: Taiwan tour agency.

a steady state that all participants are not benefited by any unilateral deviation [8]. This implies that all participants satisfy with the equilibrium outcome simultaneously. The equilibrium is applied to measure the outcome quality. For example, Yan et al. find the Nash equilibrium in the optimal bandwidth scheduling problem [9].), where the SEP's revenue is identical to that obtained in the Vickrey-Clarke-Groves auction [10]. Because most advertisers aim at maximizing the obtained utility, we treat the bidding behaviors of maximizing the utilities as the rational bidding.

However, not all strategic bidding behaviors are rational. For example, some advertisers apply the flaw of the GSP to manipulate the payments of other advertisers rather than to maximize their own utility. This kind of bidding strategies is called the vindictive bidding [2].

In the GSP, each payment equals to the next-ranked bid value. So, submitting the price between the previous-ranked and the next-ranked bid values will win the same slot if other advertisers do not update their bid values. The vindictive bidding utilizes this property to manipulate the competitor's payments. We use the example shown in Table 1 to describe the vindictive bidding. For convenience, we use a round to indicate that every time the SSA receives a keyword query. Suppose three advertisers $\left\{\operatorname{ad}_{1}, \mathrm{ad}_{2}, \mathrm{ad}_{3}\right\}$ compete for two slots $\left\{\mathrm{sl}_{1}, \mathrm{sl}_{2}\right\}$. The bids are denoted by $b_{1}, b_{2}$, and $b_{3}$, where $b_{1}>b_{2}>b_{3} \cdot \operatorname{ad}_{1}$ and $\mathrm{ad}_{2}$ are the winners of $\mathrm{sl}_{1}$ and $\mathrm{sl}_{2}$, respectively. Based on the pricing rule of the GSP, $\operatorname{ad}_{1}$ pays $b_{2}$, $\operatorname{ad}_{2}$ pays $b_{3}$, and $\operatorname{ad}_{3}$ pays zero. Supposing $b_{1}$ and $b_{3}$ are fixed, $\mathrm{ad}_{2}$ wins $\mathrm{sl}_{2}$ by proposing any price between $b_{1}$ and $b_{3}$. If $\mathrm{ad}_{2}$ bids $b_{2}^{+}$, where $b_{1}>b_{2}^{+}>b_{2}, \operatorname{ad}_{2}$ still wins $\mathrm{sl}_{2}$ and pays $b_{3}$, but the payment of $\mathrm{ad}_{1}$ is increased from $b_{2}$ to $b_{2}^{+}$. Obviously, raising the price from $b_{2}$ to $b_{2}^{+}$is a vindictive bidding. In the following context, $\mathrm{ad}_{2}$ is called as the vindictive advertiser while $\mathrm{ad}_{1}$ is the target advertiser.

The major objective of the vindictive bidding is to maximize the payment of the target advertiser. Some negative effects that resulted from the vindictive bidding are observed. For the target advertisers, the utilities are decreased because the payments are raised by the vindictive bidding. For the outcome stability, the equilibrium is eliminated in some cases [2]. In this paper, investigating the effects that resulted from the vindictive bidding is our major
TABLE 1: Example of the vindictive bidding in a SSA.

\begin{tabular}{lccc}
\hline Terms & $\mathrm{ad}_{1}$ & $\mathrm{ad}_{2}$ & $\mathrm{ad}_{3}$ \\
$\begin{array}{l}\text { Occupied slot } \\
\text { Bid price in the current }\end{array}$ & $\mathrm{sl}_{1}$ & $\mathrm{sl}_{2}$ & Nothing \\
round & $b_{2}$ & $b_{3}$ \\
$\begin{array}{l}\text { Bid price in the next } \\
\text { round }\end{array}$ & $b_{1}$ & $b_{2}^{+}($where & $\left.b_{1}>b_{2}^{+}>b_{2}\right)$ \\
\hline
\end{tabular}

objective, where the vindictive advertisers have different valuations.

The strategic bidding behaviors that we considered include the rational bidding and the vindictive bidding. According to the studies of $\mathrm{Bu}$ et al. [2], we survey three vindictive bidding behaviors: the malicious bidding, the selective bidding, and the conservative bidding. Note that the malicious, selective, and conservative advertisers represent the bidders who utilize the malicious, selective, and conservative bidding strategies to determine the bid values. The malicious advertisers always try to maximize the payments of the target advertisers. They have higher degree of the vindictiveness than other vindictive bidding advertisers. The selective advertisers bid vindictively only if they miss their desire positions. The bidding behaviors of the conservative advertisers are similar to that of the selective advertisers, but the utilities of the conservative advertisers are never sacrificed for bidding vindictively.

We study the effects of the vindictive bidding in an English auction-based SSA: the nondecreasing sponsored search auction (NDSSA) [1]. The English auction is widely applied in the real world application and the electronic marketing because of the easy implementation. Moreover, various outcomes can be realized by adjusting the auction parameters. Thus, considering the NDSSA is close to real world behaviors and helps us to understand the effects resulted from the vindictive bidding.

Increasing target advertisers' payments is the major objective of the vindictive advertisers. Obviously, the vindictive bidding increases SEP's revenue and decreases the utilities of the target advertisers. However, we are interested in the relationship between the negative effects resulted from the vindictive bidding and the valuations of the vindictive advertisers. To focus on the negative effects resulted from the vindictive bidding, we consider only one vindictive advertiser in each simulation instance. According to our experiment results, the SEP's revenue is proportional to the degree of the vindictiveness. When the advertisers have stronger vindictiveness, the SEP will gain more revenue. On the other hand, advertisers' utilities are reduced by the vindictive bidding in most instances. Only conservative behaviors improve the utilities in some instances because the utility is not sacrificed for the vindictive bidding. The advertiser with small valuation without bidding vindictively has no idea how to win any slot, but they may earn the last slot by adopting the selective bidding. The advertisers with low valuation should consider the slightly vindictive bidding strategy. In summary, even if bidding vindictively increases 
competitors' payments, vindictive advertisers may also lose a part of the utility in most instances.

\section{Related Works}

There are two English auction-based SSAs: the generalized English auction [6] and the NDSSA [1]. The generalized English auction considers full processes of the English auction to rank advertisers. When the valuations are very close to each other; the generalized English auction requires huge time to determine ranking results. In real world SSAs, most Internet users do not wait to receive a web page for a long time. Therefore, the NDSSA is more appropriate than the generalized English auction in practice because the NDSSA utilizes the rank-by-bid strategy to determine the ranking results.

Many strategic bidding strategies are studied. For example, the forward-looking bidding strategy proposed by Bu et al. [4], the multibidding strategy proposed by Bu et al. [11], the balanced bidding strategy proposed by Cary et al. [5], and some vindictive bidding strategies [2]. The bidding strategies can be classified by the rational bidding and the vindictive bidding. The rational bidding aims to maximize the own utility while the vindictive bidding focuses on maximizing the competitors' payments.

In the SSAs with complete information, the SEP can utilize some mechanisms to prevent the negative effects resulted from the vindictive bidding. For example, the VickreyClarke-Groves auction guarantees the incentive compatibility, so the vindictive bidding is the dominated strategy [1214]. However, not all information in the real world SSAs is common knowledge. Most advertisers do not expose their valuations and adopted bidding strategies to other advertisers and the SEP. So, it is hard to capture the vindictive advertisers. Moreover, the effects resulted from the vindictive advertisers with various valuations and the interactions of the advertisers adopting different bidding strategies are still unclear.

\section{Preliminaries}

3.1. Auction Model. Consider a repeated SSA which focuses on a specific keyword. The search result page consists of $K$ advertising slots. Each slot $\mathrm{sl}_{j}$ includes a click-throughrate (CTR) $\theta_{j}$ which is the click probability that the Internet users click the advertisement on $\mathrm{sl}_{j}$. We assume that the slot rearranged in a better position will receive more clicks; that is, $\theta_{1}>\theta_{2}>\cdots>\theta_{K}$. Suppose $N$ advertisers participate in the auction, where $K<N$. As a keyword query arrives, each advertiser $\operatorname{ad}_{i}$ submits a bid $b_{i}$ to declare the maximum payment per click. When an Internet user clicks the advertising link of $\mathrm{ad}_{i}, \operatorname{ad}_{i}$ receives the income $v_{i}$ which is named as the valuation. We assume that $\mathrm{ad}_{i}$ wins $\mathrm{sl}_{i}$. According to the pricing rule of the GSP, the payment of $\mathrm{ad}_{i}$ denoted by $p_{i}$ is equal to the next-ranked bid price; that is, $p_{i}=b_{i+1}$. Given the bid profile $b=\left\{b_{1}, b_{2}, \ldots, b_{N}\right\}$, the expected utility of $\operatorname{ad}_{i}$ is $u_{i}(b)=\left(v_{i}-p_{i}\right) \times \theta_{i}$. SEP's expected revenue comes from the sum of advertisers' payments, that is, $\sum_{s=1}^{K} b_{s+1} \times \theta_{s}$.
In the real world applications, the valuations and the bidding strategies adopted by advertisers are private information. Thus, the SEP or the advertisers do not know who is the vindictive advertiser. Note that the bid profile and the payment profile are public only if the auction result is announced. This implies that each advertiser does not access the bid values of other advertisers when computing a new bid price.

3.2. Nondecreasing Sponsored Search Auction. The NDSSA is an English auction-based mechanism [1]. Similar to the English auction, the NDSSA considers the minimum increase price (MIP) to indicate the lower bound of the bid value for all updated bids. The SEP should determine two parameters of the NDSSA: an initial MIP value and an MIP adjusting strategy. The initial MIP value indicates the minimum bid increment in the second round (there is no restriction for submitting the bid price in the first round). The MIP adjusting strategy recomputes the MIP value that will be applied in the next round after obtaining the auction result in each round.

The MIP restricts the bid prices. Submitting the following bid prices is allowed.

Rule 1 . The same value as that proposed in the last round.

Rule 2. A price higher than the last one plus the MIP value.

To focus on the effects resulted from the vindictive bidding, we consider the fixed-MIP as our MIP adjusting strategy. The fixed-MIP uses the initial MIP value throughout the auction. In other words, the MIP is not changed until the NDSSA is restarted every day.

When an Internet user issues a keyword query, the NDSSA selects $N$ advertisers as the candidates for advertising. Each candidate is asked to propose the bid value $b_{i}$ which is verified by the MIP. We assume that all bids are legal. Then, all candidates are ranked in the decreasing order of the bid values. First $K$ highest candidates are claimed as the winners. Eventually, the allocation result and the new MIP value will be announced to each advertiser. Note that we consider the fixed-MIP, each advertiser knows the MIP setting in each round. If the SEP adopts the other MIP adjusting strategies, the new MIP is announced in the end of each round.

If we follow the bidding restriction applied in [1], some vindictive bidding behaviors are restricted. For example, the price difference between the current bid value and the previous-ranked bid value is smaller than the MIP value. The vindictive advertiser can only bid the price higher than that proposed by the previous-ranked advertiser. This bid update is not the vindictive bidding. So, the third feasible bid price is considered.

Rule 3. Each $\mathrm{ad}_{i}$ has an opportunity to raise the bid without the MIP constraint, but $\mathrm{ad}_{i}$ cannot update the bid price in the future.

\section{Bidding Behaviors}

According to the aspect of the game theory [8], rational advertisers aim at maximizing the obtained utility. However, 
the vindictive advertisers prefer to increase the payments of the target advertisers. Based on the studies on $[2,4]$, we consider a rational bidding and three vindictive bidding behaviors. If the vindictive advertiser wins the top slot, that is, he/she does not have any competitor, the bid price will not be updated in the next round.

4.1. Rational Advertiser. Rational advertisers try to maximize the expected utility $u_{i}(b)$. The bid price is updated only if winning the previous-ranked slot improves $u_{i}(b)$. For example, the forward-looking bidding behaviors [4] and the greedy bidding strategies [5] are rational bidding. Given the MIP value $x$, the bidding behavior of the rational advertiser in the NDSSA is shown in equality (1) where $b_{-i}=\left\{b \backslash b_{i}\right\}, \forall i>$ 1. If winning $\mathrm{sl}_{i-1}$ results in higher utility, $\operatorname{ad}_{i}$ will bid submit a higher price, and $b_{i}$ otherwise:

$$
\begin{aligned}
& \mathrm{BH}_{i}^{r}(b) \\
& \quad= \begin{cases}b_{i}, & \text { if } u_{i}\left(b_{i-1}+0.1, b_{-i}\right) \leq u_{i}(b) \\
\max \left(b_{i-1}+0.1, b_{i}+x\right), & \text { otherwise. }\end{cases}
\end{aligned}
$$

4.2. Selective Advertiser. Selective advertisers retaliate against the target advertiser only if the desire position is not obtained. When $\mathrm{ad}_{i}$ is benefited by winning the previous-ranked slot but the MIP is too high to update the bid price, that is, $b_{i}+x>v_{i}$, a vindictive action takes place. The bid value is increased to $\left(b_{i-1}-0.1\right)$ to maximize the payment of the target advertiser. When $v_{i-1}<v_{i}, \mathrm{ad}_{i}$ can propose a higher bid value than $b_{i-1}$ if the previous-ranked slot is benefited to $\mathrm{ad}_{i}$. So, $v_{i-1}>v_{i}$ is the essential condition for the selective bidding strategies. The selective bidding is defined in equality (2) for each $i>1$.

$$
\mathrm{BH}_{i}^{s}(b)= \begin{cases}b_{i-1}-0.1, & \text { if } u_{i}\left(b_{i-1}+0.1, b_{-i}\right)>u_{i}(b), \\ & b_{i}+x>v_{i}, \quad v_{i-1}>v_{i} \\ \mathrm{BH}_{i}^{r}(b), & \text { otherwise. }\end{cases}
$$

4.3. Conservative Advertiser. Selective advertisers may receive negative utility if $\mathrm{BH}_{i}^{s}(b)=b_{i-1}-0.1>v_{i}$. The conservative bidding strategy does not sacrifice the utility for the vindictive bidding. This implies that the conservative advertisers do not overbid, and the utility can be protected. The conservative bidding strategy is formulated in the following equality:

$$
\mathrm{BH}_{i}^{c}(b)= \begin{cases}\min \left\{b_{i}-0.1, v_{i}\right\}, & \text { if } u_{i}\left(b_{i-1}+0.1, b_{-i}\right)>u_{i}(b) \\ & b_{i}+x>v_{i} \\ \mathrm{BH}_{i}^{r}(b), & \text { otherwise. }\end{cases}
$$

4.4. Malicious Advertiser. The malicious advertiser $\mathrm{ad}_{i}$ is most vindictive than above advertisers. The objective of the malicious advertisers is to maximize the payments of the target advertiser without respect to the own utility. So, $\mathrm{ad}_{i}$ always bids $\left(b_{i-1}-0.1\right)$. The bid price of the malicious advertiser $\mathrm{BH}_{i}^{m}(b)$ is shown in the equality

$$
\mathrm{BH}_{i}^{m}(b)=b_{i-1}-0.1, \quad \forall i>1 .
$$

\section{Experiments}

5.1. Parameters Settings. We consider $K=6$ and $N=9$, and no advertiser will depart from the auction. Referring to the experiment settings in [3], the valuation instances are determined by the uniform distribution, triangular distribution, normal distribution, exponential distribution, and Pareto distribution within 1 and 50. The valuations are sorted in the decreasing order in each instance. We generate five initial bid profiles by four normal distributions with different standard deviation and one random distribution. We use Zipf's distributions [3] to generate CTR instances (from the studies of $[3,15]$, most click behaviors can be simulated by Zipf's distribution and the geometrically decreasing sequence with different parameters. For the big value of $\alpha$, the CTR gap between two successive slots is smaller than that of the small value of $\alpha$. This implies that the bigger values of $\alpha$ result in the keywords with lower market value, and vice versa). Given the parameter $\alpha$, where $\alpha \epsilon$ $\mathscr{R}^{+}$, the CTR value under Zipf's distribution is shown in the equality

$$
\theta_{j} \propto \frac{1}{j^{\alpha}}, \quad \forall \alpha \in \mathscr{R}^{+} .
$$

The initial MIP values are set from 1 to 49 with the fixedMIP strategy. Because the maximum valuation is 50 in our simulations, no advertiser can update bid prices if the initial MIP value is higher than 50 . The maximum initial MIP value is set to be 49 to prevent the case that all advertisers can not update bids throughout the auction.

We observe the relationship between the effects resulted from the vindictive bidding and the valuations of the vindictive advertisers. The advertisers with higher valuations win a slot in high probability than the advertisers with lower valuations. Consider that the vindictive advertisers having different valuations are essential. In our simulations, we assume that each advertiser is a vindictive bidder in each auction. Therefore, each auction is performed ten times (including one case with all rational advertisers and nine cases that one vindictive advertiser is in each case).

5.2. Search Engine Revenue. We first evaluate the SEP's revenue under the vindictive bidding consideration. To simplify the problem scale, we consider one vindictive advertiser in each instance. The result is shown in Figure 2. The SEP's revenue is averaged by the results captured from all combinations of valuation, CTR, and initial bid prices. The horizontal axis denotes the index of the vindictive advertiser, and the vertical axis represents the SEP's revenue. The benchmark is the SEP's revenue obtained when all advertisers are rational. 


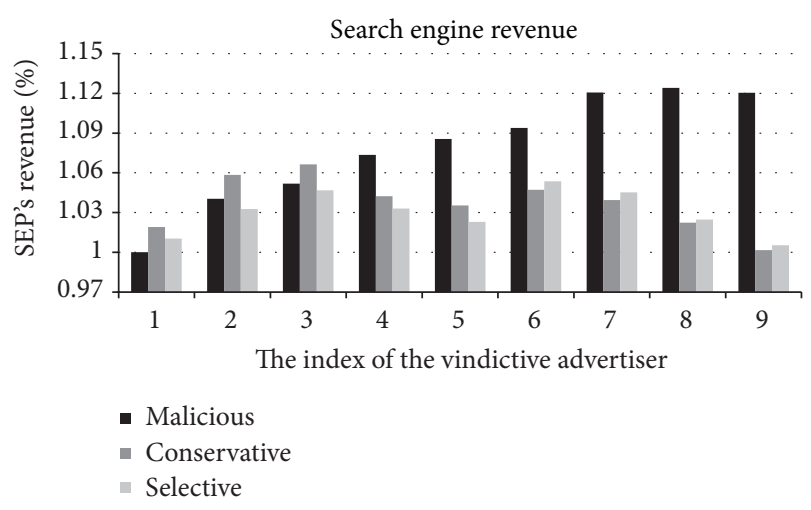

FIgURE 2: The comparison between the rational and the vindictive advertisers for SEP's revenue.

Observation 1. The SEP's revenue is inversely proportional to the valuation of the malicious advertiser.

The malicious advertiser always retaliates against the target advertiser. In the worst case, the malicious advertiser occupies the next-ranked slot of the advertiser with maximum valuation. Because any vindictive behavior will not bid higher than the previous-ranked bid price, the bid update is stopped at the second slot. Therefore, the malicious advertiser with lower valuation produces higher SEP's revenue.

Observation 2. The malicious advertiser with highest valuation does not vary the SEP's revenue.

When the malicious advertiser wins the first slot, no advertiser is in front of the first slot. The vindictive advertiser will not raise his/her bid price. Thus, SEP's revenue is not varied by the malicious advertiser with the highest valuation.

Observation 3. For the winners, the SEP gains more revenue from conservative advertisers than selective advertisers. The opposite results occur for losers.

The bid value of the conservative advertiser is bound by the valuation, while it is not considered by the selective advertisers. The selective losers, that is, $\operatorname{ad}_{7}, \operatorname{ad}_{8}$, and $\mathrm{ad}_{9}$, result in higher SEP revenue than conservative losers because no loser can win the target slot. The selective losers continuously retaliate to the sixth winner even though overbidding, but this is not considered by the conservative advertisers. Then, the SEP gains more revenue from selective losers than conservative losers.

For the winners, the SEP receives more revenue from the conservative advertisers than the selective advertisers. Because the previous-ranked advertiser may have a lower valuation than the selective advertiser, the selective advertisers do not bid vindictively frequently. So, the conservative winners contribute more SEP's revenue than the selective winners.

5.3. Utility of Vindictive Advertisers. Next, we focus on the relationship between advertisers' utilities and the valuation of the vindictive advertiser. We use the ratio of the vindictive advertiser's utility to that of the rational advertiser. The results obtained from the malicious, selective, and conservative advertisers are shown in Figures 3(a), 3(b), and 3(c), respectively. In each figure, the horizontal axis denotes the index of the vindictive advertiser. The vertical axis represents the ratio of the instances that the vindictive advertiser's utility is better than, the same as, or worse than that of the rational advertiser. In Figure 3(a), for example, the third advertiser has more utility by adopting the malicious bidding than the rational bidding in $20 \%$ of the instances.

Observation 4. Malicious advertisers are benefited in only a few instances (20\% instances at most).

The objective of the malicious advertisers is to maximize the payments of the target advertisers without caring about their own utilities. So, the utility reduce is in expectation. The result in Figure 3(a) matches that in Figure 2. Since the SEP's revenue completely comes from the sum of advertisers' payments, the SEP is benefited when the advertiser's utility is decreased, and vice versa.

Observation 5. In most instances, the utility of the selective winners is equal to that of the rational advertiser. On the other hand, the selective losers receive more utility than the selective winners in high probability.

Similar to the discussion in Observation 3, the selective winners and the rational winners obtain the same utility in more than $60 \%$ of instances. Since the NDSSA considers the GSP as the pricing strategy, the selective advertiser's valuation may be higher than that of the target advertiser. Therefore, the vindictive bidding does not occur frequently. The selective losers continuously propose vindictive bid values because the target slot is not obtained. When the selective losers and the target advertiser raise prices and move forward a slot simultaneously, the selective loser obtains the last slot. So, the utility of the selective loser is improved. Eventually, the selective losers have more opportunities than rational losers to win the target slots.

Observation 6. The advertiser's utility is slightly improved by the conservative bidding.

The bid value of the conservative advertiser is bound by the valuation. The overbidding does not take place, so the utility of the conservative advertiser is better than that of the malicious advertiser. Therefore, slightly bidding vindictively improves the advertiser's utility in some instances, and no overbidding is necessary for improving the utility under the vindictive bidding consideration.

In summary, bidding vindictively without turning back results in high payment increment for the target advertiser. But, the utilities of the malicious advertisers are also be decreased by their vindictive bidding behaviors. The selective advertisers can save the utilities in most instances. For the conservative advertisers, although the utility is protected, the auction results are hard to be predicted in terms of the utility. 


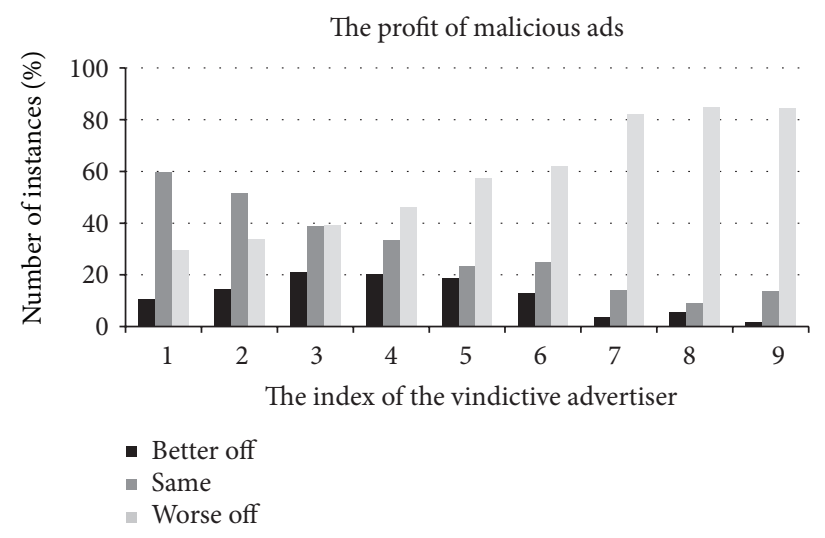

(a) The utility comparison of the malicious advertisers

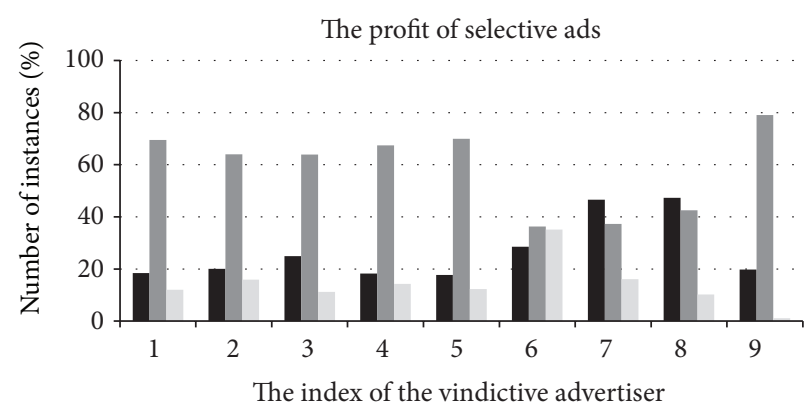

- Better off

- Same

- Worse off

(b) The utility comparison of the selective advertisers

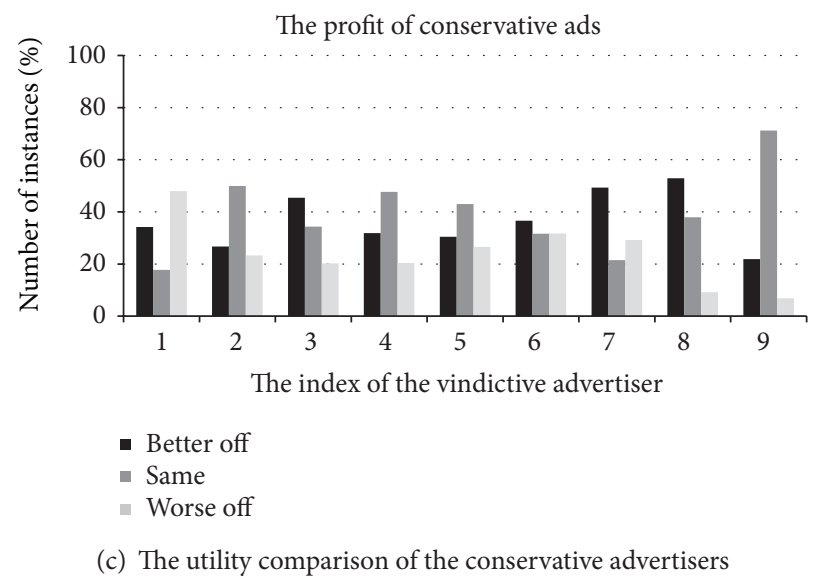

FIgure 3: Advertisers' utilities obtained by different vindictive bidding strategies.

5.4. The Effects Resulted from the Vindictive Bidding in Different Keywords. In this experiment, we observe the relationship between different keyword phrases and the SEP's revenue when one vindictive advertiser is considered. The simulation results are shown in Figure 4. The horizontal axis denotes the CTR distributions that are produced by different values of $\alpha$. Higher value of $\alpha$ results in smaller CTR gap between two successive slots and that represents the keyword phrases with lower market value. The vertical axis represents the SEP's revenue which is normalized by that obtained from the rational bidders. The winners are drawn by solid lines while the losers are illustrated by the dashed lines. Although the sixth advertiser is still a winner, we use the dashed line to represent it because he/she is affected by the vindictive losers.

Observation 7. For the losers, the improvement of the SEP's revenue in the keywords with high market value is much more than that with low market value. The opposite result is obtained for the winners.

Form Figure 4, the tendencies of the losers' curves are descending. However, the tendencies of the winners' curves are not varied dramatically except for the second advertiser. The instances with small values of $\alpha$ represent the keywords with high market values. So, in the last slot, the CTR with the small value of $\alpha$ is higher than that with high value of $\alpha$, and vice versa. In other words, the last slot with small value of $\alpha$ is more valuable than that with high value of $\alpha$. Therefore, it is reasonable that the improvement of the SEP's revenue for the keywords with high market value is higher than that for the keywords with low market value.

On the other hand, the variance of the revenue improvement for the SEP is small for most vindictive advertisers. This implies that different keyword phrases do not vary the revenue improvement for the SEP too much. Because the objective of the vindictive bidding is to maximize the payments of the target advertiser. The price of the vindictive bidding depends on the bid value proposed by the target advertiser. So, the relationship between the keyword phrases and the revenue improvement of the SEP is not very close for the winners.

5.5. The Effects Resulted from the Vindictive Bidding in Different Minimum Increase Prices. In this simulation, we evaluate the relationship between the initial MIP settings and the SEP's revenue. The simulation result is illustrated in Figure 5. The horizontal axis denotes the initial MIP setting. The vertical 


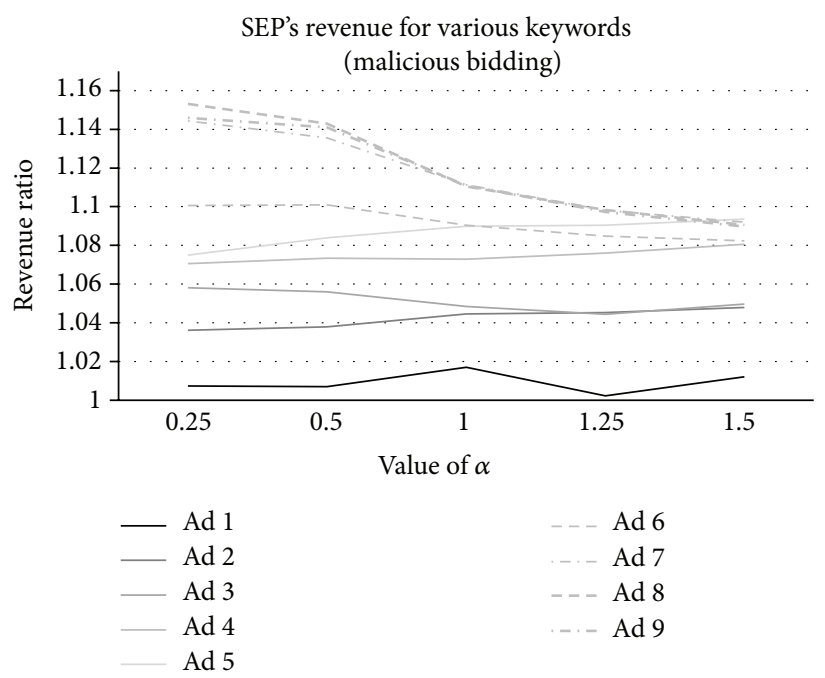

(a) The revenue variance of the SEP for one malicious advertiser

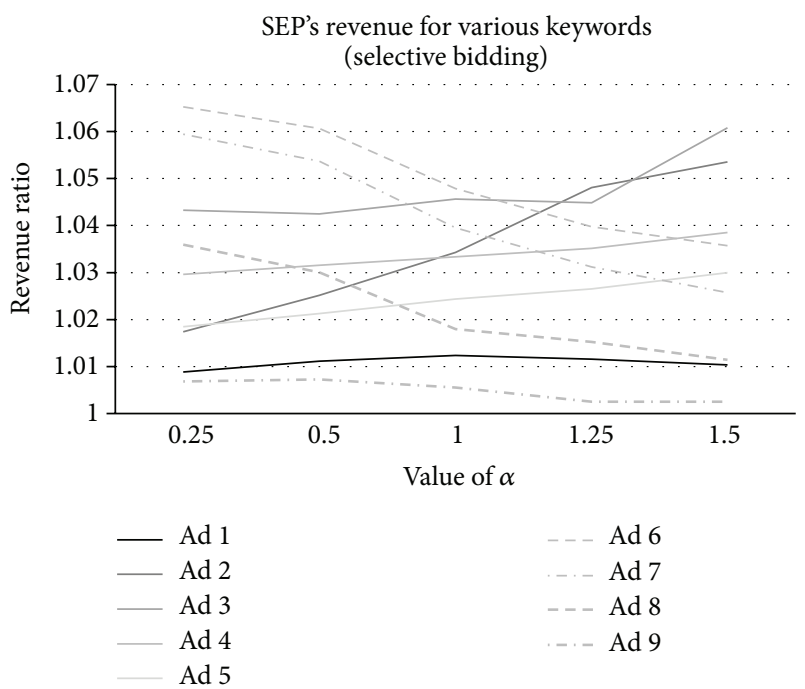

(b) The revenue variance of the SEP for one selective advertiser

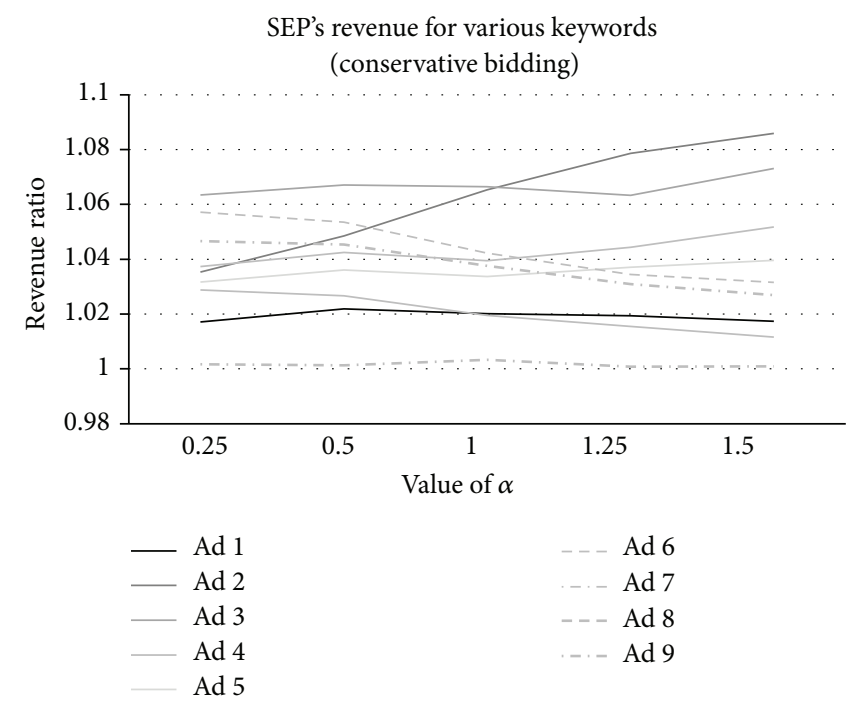

(c) The revenue variance of the SEP for one conservative advertiser

FIGURE 4: SEP's revenue comparisons with different vindictive bidding strategies for various keyword phrases.

axis represents the SEP's revenue which is normalized by that obtained from the rational advertisers.

Observation 8. The amount of the SEP's revenue raised by the malicious advertisers is higher than that resulted from other vindictive advertisers.

From Observations 1 and 2, the improved amount of the SEP's revenue is proportional to the degree of the vindictiveness. Therefore, the SEP will receive more revenue when the vindictive advertiser adopts the bidding strategy with stronger vindictiveness.

Observation 9. Before the initial MIP 42, the SEP's revenue is proportional to the initial MIP values. The opposite results are derived as the initial MIP is bigger than 42 .
By increasing the initial MIP value, the bidding competition is raised. Simultaneously, the vindictiveness is also raised. So, the improvement of the SEP's revenue is raised as increasing the initial MIP setting.

From Figure 5, the revenue improvement is at a watershed in the initial MIP 42. According to our input data, the maximum gap between the valuation and the initial bid price is 44 . This implies that no advertiser can update the bid value if the initial MIP is set to be higher than 44. So, it is rational that the watershed of the SEP's revenue presents before the initial MIP 44. However, the vindictive advertiser still can bid vindictively by the third bidding restriction that we relaxed in Section 3.2. So, this is why the SEP's revenue is more than that obtained by rational bidders in the initial MIP values higher than 42 . 


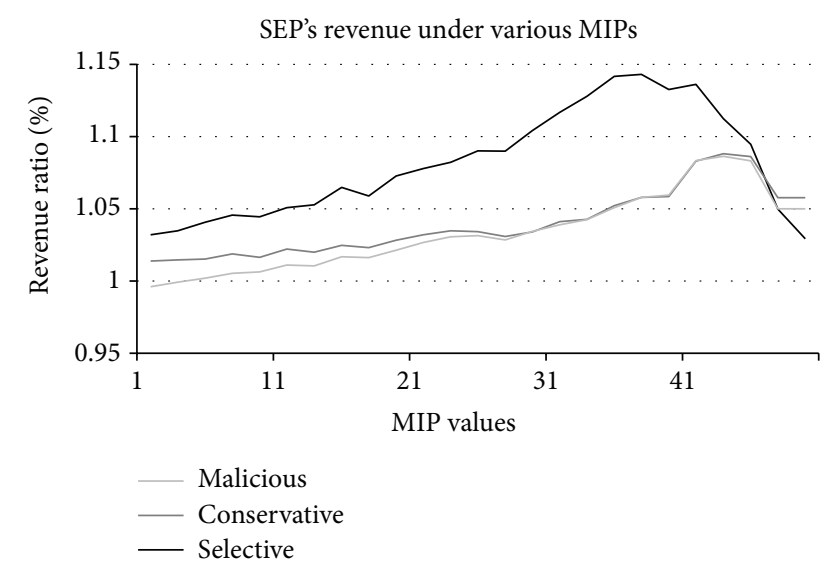

FIGURE 5: The revenue comparison for the SEP given various initial MIP settings.

\section{Conclusion}

The vindictive bidding occurs frequently in the real world SSAs. We survey three well-defined vindictive bidding strategies and one rational bidding behavior. Our major objective is to analyze the effects resulted from the vindictive bidding for the aspects of the advertisers and the SEP, where the vindictive advertisers have different valuations. According to our simulation results, the SEP is benefited by all kinds of vindictive bidding behaviors. This means that rational advertiser's payment is increased by the vindictive bidding. On the other hand, the degree of the vindictiveness affects the advertiser's utility. The utility of the advertiser with high vindictiveness is reduced in most instances. Advertisers' utilities are improved in some instances only if submitting the bids with slightly vindictive prices. Therefore, even though vindictive bidding increases the competitor's payment, the advertisers adopting vindictive bidding behaviors also lose a part of the utilities.

The effects of vindictive bidding behaviors are studied in this paper. In our simulation, we notice that the advertiser is benefited by slightly vindictive bidding behavior in some situations. Understanding the conditions where vindictive bidding behavior is beneficial for the advertiser's utility is our next objective.

\section{Acknowledgments}

This work was supported in part by Taiwan NSC under Grants no. NSC 102-2221-E-274 -004 and NSC 102-2221-E-194-054-. The author would like to thank reviewers for their insightful comments which helped to significantly improve the paper.

\section{References}

[1] C. Tsung, H. Ho, and S. Lee, "Analysis of stable prices in non-decreasing spon- sored search auction," in Proceedings of the 13th International Workshop on Agent-Mediated Electronic Commerce, pp. 84-96, 2011.
[2] T.-M. Bu, L. Liang, and Q. Qi, "On robustness of forwardlooking in sponsored search auction," Algorithmica, vol. 58, no. 4, pp. 970-989, 2010.

[3] M. Naldi, G. D'Acquisto, and G. F. Italiano, "The value of location in keyword auctions," Electronic Commerce Research and Applications, vol. 9, no. 2, pp. 160-170, 2010.

[4] T.-M. Bu, X. Deng, and Q. Qi, "Forward looking Nash equilibrium for keyword auction," Information Processing Letters, vol. 105, no. 2, pp. 41-46, 2008.

[5] M. Cary, A. Das, B. Edelman et al., "Greedy bidding strategies for keyword auctions," in Proceedings of the 8th ACM Conference on Electronic Commerce (EC '07), pp. 262-271, June 2007.

[6] B. Edelman, M. Ostrovsky, and M. Schwarz, "Internet advertising and the generalized second-price auction: selling billions of dollars worth of keywords," American Economic Review, vol. 97, no. 1, pp. 242-259, 2007.

[7] Y. Narahari, D. Garg, R. Narayanam, and H. Prakash, Game Theoretic Problems in Network Economics and Mechanism Design Solutions, Springer, 2009.

[8] N. Nisan, T. Roughgarden, E. Tardos, and V. V. Vazirani, Algorithmic Game Theory, Cambridge University Press, 2007.

[9] X. Yan, H. Li, L. Wang, and P. Shen, "Optimal bandwidth scheduling of networked learning control system based on mash theory and auction mechanism," Mathematical Problems in Engineering, vol. 41, no. 4, pp. 617-631, 2013.

[10] H. R. Varian, "Position auctions," International Journal of Industrial Organization, vol. 25, no. 6, pp. 1163-1178, 2007.

[11] T.-M. Bu, X. Deng, and Q. Qi, "Multi-bidding strategy in sponsored search auctions," Journal of Combinatorial Optimization, vol. 23, no. 3, pp. 356-372, 2012.

[12] W. Vickrey, "Counterspeculation, auctions, and competitive sealed tenders," Journal of Finance, vol. 16, no. 1, pp. 8-37, 1961.

[13] E. H. Clarke, "Multipart pricing of public goods," Public Choice, vol. 11, no. 1, pp. 17-33, 1971.

[14] T. Groves, "Incentives in teams," Econometrica, vol. 41, no. 4, pp. 617-631, 1973.

[15] J. Feng, H. K. Bhargava, and D. M. Pennock, "Implementing sponsored search in web search engines: computational evaluation of alternative mechanisms," INFORMS Journal on Computing, vol. 19, no. 1, pp. 137-148, 2007. 


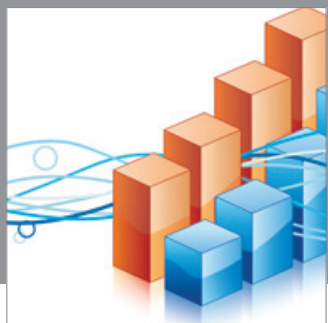

Advances in

Operations Research

mansans

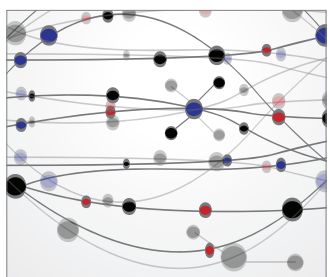

The Scientific World Journal
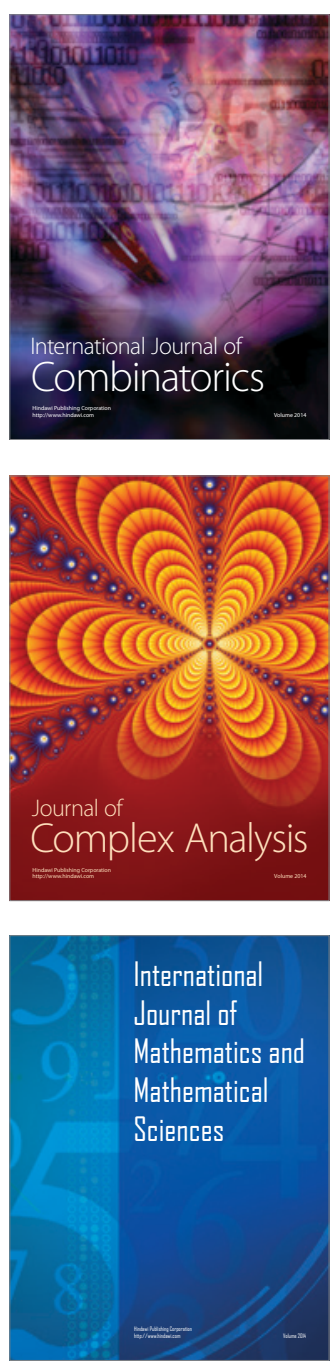
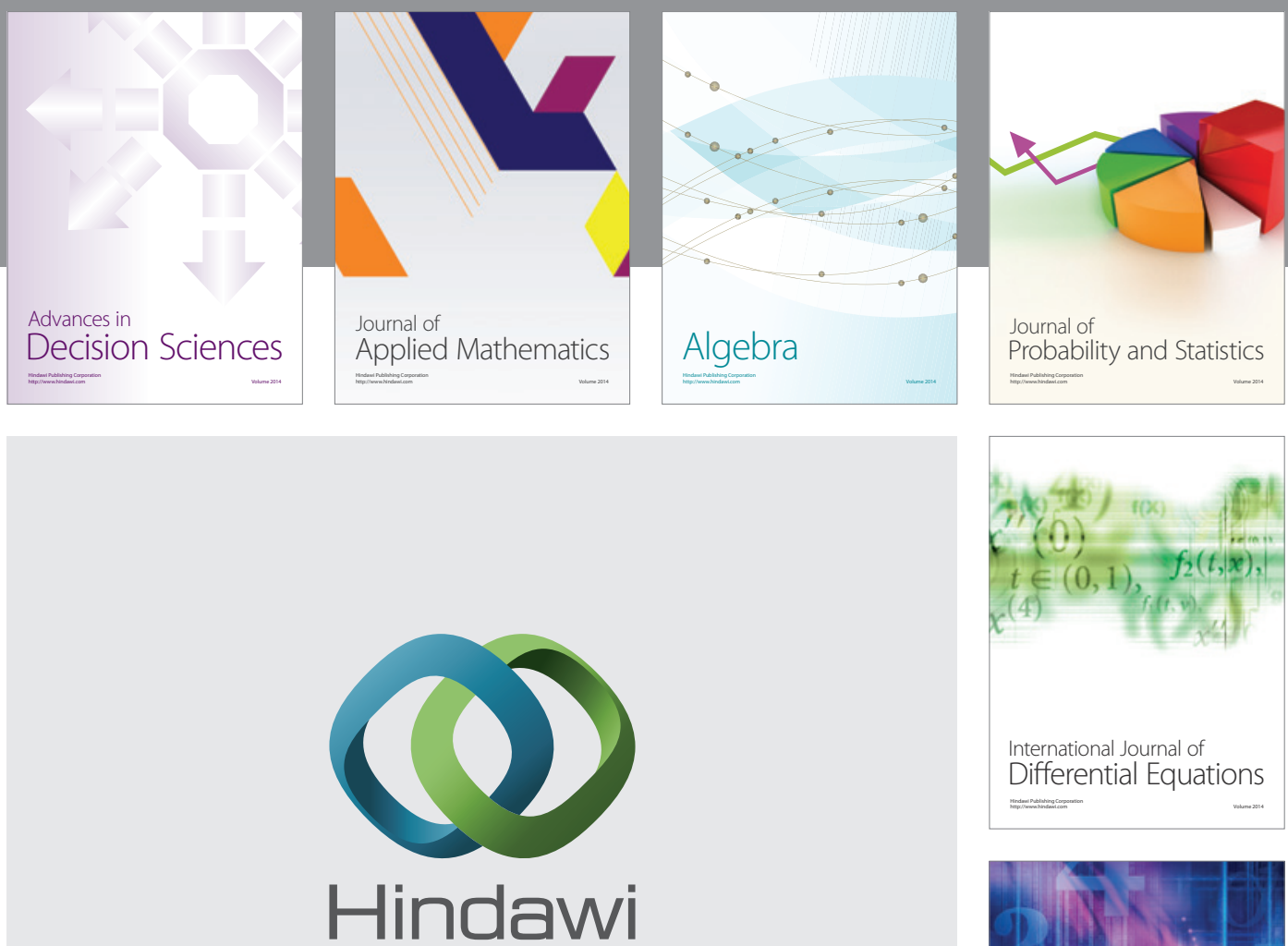

Submit your manuscripts at http://www.hindawi.com
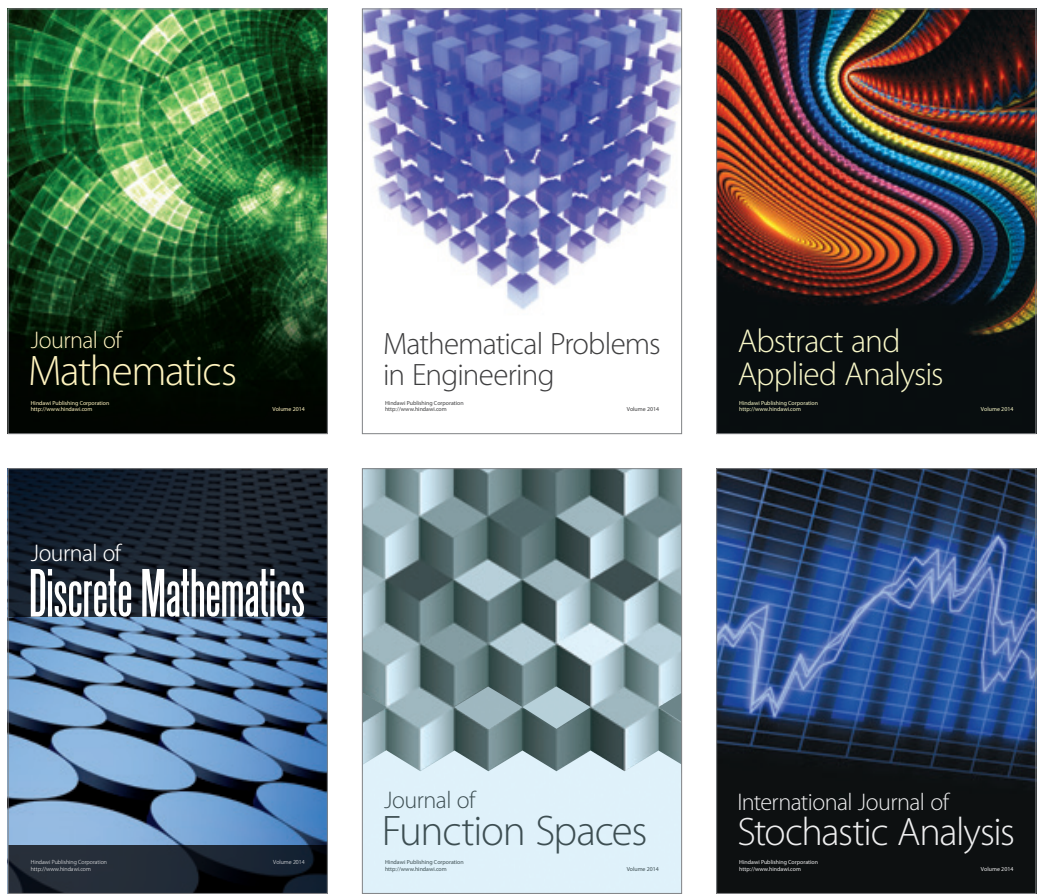

Journal of

Function Spaces

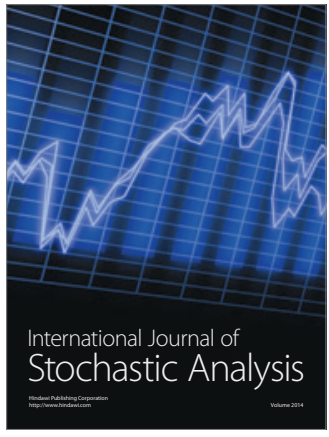

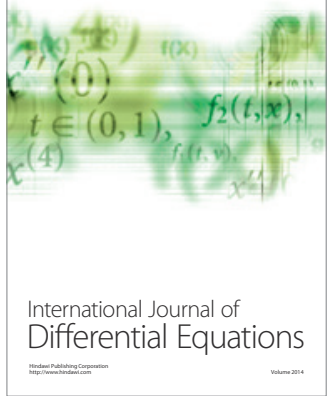
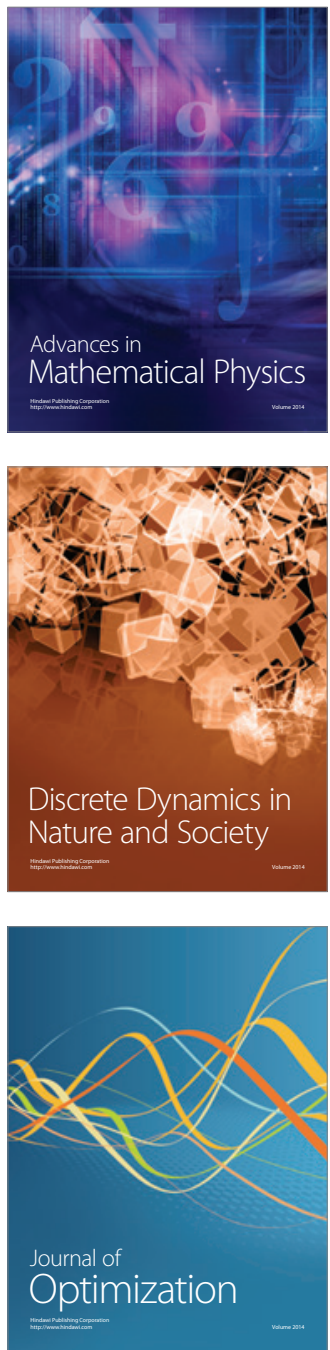